\title{
Sufficient conditions for univalence obtained by using Briot-Bouquet differential subordination
}

\author{
Georgia Irina Oros*, Alina Alb Lupaş \\ Department of Mathematics and Computer Science, University of Oradea, Romania
}

Received November 02, 2019; Revised December 19, 2019; Accepted December 25, 2019

Copyright (C) 2020 by authors, all rights reserved. Authors agree that this article remains permanently open access under the terms of the Creative Commons Attribution License 4.0 International License

\begin{abstract}
In this paper, we define the operator $I^{m}: \mathcal{A} \rightarrow \mathcal{A}, I^{m}[f](z)=(1-\lambda) S^{m}[f](z)+\lambda L^{m}[f](z), z \in U$, differential-integral operator, where $S^{m}$ is Sălăgean differential operator and $L^{m}$ is Libera integral operator. By using the operator $I^{m}$ the class of univalent functions denoted by $M(m, \beta, \lambda), 0 \leq \beta<1,0 \leq \lambda \leq 1, m \in \mathbb{N}$ is defined and several differential subordinations are studied. Even if the use of linear operators and introduction of new classes of functions where subordinations are studied is a well-known process, the results are new and could be of interest for young researchers because of the new approach derived from mixing a differential operator and an integral one. By using this differential-integral operator, we have obtained new sufficient conditions for the functions from some classes to be univalent. For the newly introduced class of functions, we show that is it a class of convex functions and we prove some inclusion relations depending on the parameters of the class. Also, we show that this class has as subclass the class of functions with bounded rotation, a class studied earlier by many authors cited in the paper. Using the method of the subordination chains, some differential subordinations in their special Briot-Bouquet form are obtained regarding the differential-integral operator introduced in the paper. The best dominant of the Briot-Bouquet differential subordination is also given. As a consequence, sufficient conditions for univalence are stated in two criteria. An example is also illustrated, showing how the operator is used in obtaining Briot-Bouquet differential subordinations and the best dominant.
\end{abstract}

Keywords Analytic Function, Differential Operator, Integral Operator, Convex Function, Univalent Function, Differential Subordination, Differential Subordination Briot-bouquet, Dominant, Best Dominant

2010 AMS Mathematics Subject Classification : 30C80, 30C45.

\section{Introduction and Preliminaries}

The concept of differential subordinations was introduced in two papers in 1978 and 1981 ([8], [9]) by S.S. Miller and P.T. Mocanu and developed later by many authors in numerous published papers ([2], [12], [13], [14], [15]).

Next we give the main classes of univalent functions used in the paper.

Let $U$ denote the unit disk of the complex plane: $U=\{z \in \mathbb{C}:|z|<1\}$.

Let $\mathcal{H}(U)$ be the space of holomorphic functions in $U$, and let

$$
\mathcal{A}_{n}=\left\{f \in \mathcal{H}(U): f(z)=z+a_{n+1} z^{n+1}+\ldots, z \in U\right\},
$$

with $\mathcal{A}_{1}=\mathcal{A}$, 
be the class of holomorphic and univalent functions in the open unit disk $U$, with conditions $f(0)=0, f^{\prime}(0)=1$, that is the holomorphic and univalent functions with the following power series development $f(z)=z+a_{2} z^{2}+a_{3} z^{3}+\ldots, z \in U$.

For $a \in \mathbb{C}$ and $n \in \mathbb{N}^{*}$, denote by

$$
\begin{gathered}
\mathcal{H}[a, n]=\left\{f \in \mathcal{H}(U): f(z)=a+a_{n} z^{n}+a_{n+1} z^{n+1}+\ldots, z \in U\right\}, \\
\mathcal{K}=\left\{f \in \mathcal{A}: \operatorname{Re}\left(\frac{z f^{\prime \prime}(z)}{f^{\prime}(z)}+1\right)>0, z \in U\right\}
\end{gathered}
$$

the class of normalized convex functions in $U$ and

$$
\mathcal{S}^{*}=\left\{f \in \mathcal{A}: \operatorname{Re} \frac{z f^{\prime}(z)}{f(z)}>0, z \in U\right\}
$$

the class of normalized starlikeness functions in $U$.

We now remind the definition of the subordination as it is given in the most important monograph related to the differential subordination method or the admissible function method which contains its fundamental notions, published in 2000 ([10]).

Definition 1. (Subordination [10, p. 4]). If $f$ and $g$ are analytic functions in $U$, then $f$ is subordinate to $g$, written $f \prec g$, or $f(z) \prec g(z)$, if there is a function $w$ analytic in $U$, with $\omega(0)=0,|\omega(z)|<1$, for all $z \in U$, such that $f(z)=g(\omega(z))$, for $z \in U$. If $g$ is univalent then $f \prec g$, or $f(z) \prec g(z)$ if and only if $f(0)=g(0)$ and $f(U) \subset g(U)$.

Definition 2. (Second-order differential subordination [10, p. 7]). Let $\psi: \mathbb{C}^{3} \times U \rightarrow \mathbb{C}$ and let $h$ be univalent in $U$. If $p$ is analytic and satisfies the (second-order) differential subordination

$$
\psi\left(p(z), z p^{\prime}(z), z^{2} p^{\prime \prime}(z) ; z\right) \prec h(z), z \in U,
$$

the $p$ is called a solution of the differential subordination. The univalent function $q$ is called a dominant of the solutions of the differential subordination or more simply a dominant, if $p \prec q$ for all $p$ satisfies $(i)$. A dominant $\widetilde{q}$ that satisfies $\widetilde{q} \prec q$ for all dominants $q$ of $(i)$ is said to be the best dominant of (i). (Note that the best dominant is unique up to a rotation of $U$ ).

If we require the more restrictive condition $p \in \mathcal{H}[a, n]$, then $p$ will be called an $(a, n)$-solution, $q$ an $(a, n)$-dominant and $\widetilde{q}$ the best $(a, n)$-dominant.

Definition 3. (Briot-Bouquet differential subordination [10, p. 80]). Let $\beta, \gamma \in \mathbb{C}, \beta \neq 0$, and let $h$ be a univalent function in $U$, with $h(0)=a$, and let $p \in \mathcal{H}[a, n]$, satisfy

$$
p(z)+\frac{z p^{\prime}(z)}{p(z)} \prec h(z), \quad z \in U .
$$

This first-order differential subordination is called the Briot-Bouquet differemtial subordination.

The name derives from the fact that a differential equation of the form

$$
q(z)+\frac{z q^{\prime}(z)}{\beta q(z)+\gamma}=h(z)
$$

is called a differential equation of Briot-Bouquet type [5, p. 403].

The original results shown in the present paper are obtained using the well-known Sălăgean differential operator which was introduced in 1983 ([18]) and is widely used by researchers in the field of Geometric Function Theory.

Definition 4. ([18]). For $f \in \mathcal{A}, m \in \mathbb{N}=\{0,1,2, \ldots\}$ the differential operator $S^{m}: \mathcal{A} \rightarrow \mathcal{A}$, is defined by

$$
\begin{aligned}
& S^{0}[f](z)=f(z), \\
& S^{1}[f](z)=z\left(S^{0}[f](z)\right)^{\prime}=z f^{\prime}(z), \\
& \vdots \\
& S^{m+1}[f](z)=z\left(S^{m}[f](z)\right)^{\prime}, z \in U .
\end{aligned}
$$

Remark 5. If $f \in \mathcal{A}$ and $f(z)=z+\sum_{k=2}^{\infty} a_{k} z^{k}$, then

$$
S^{m}[f](z)=z+\sum_{k=2}^{\infty} k^{m} a_{k} z^{k}, \quad z \in U .
$$


The other operator used for introducing the new differential-integral operator in the Main Results part is the following operator defined based on the Libera operator.

Definition 6. ([13]). For $f \in \mathcal{A}, m \in \mathbb{N}=\{0,1,2, \ldots\}, L^{m}: \mathcal{A} \rightarrow \mathcal{A}$ is defined by

$$
\begin{aligned}
& L^{0}[f](z)=f(z) \\
& L^{1}[f](z)=\frac{2}{z} \int_{0}^{z} L^{0}[f](t) d t=\frac{2}{z} \int_{0}^{z} f(t) d t \\
& \vdots \\
& L^{m}[f](z)=\frac{2}{z} \int_{0}^{z} L^{m-1}[f](t) d t .
\end{aligned}
$$

In the same paper ([13]), the following result related to the operator in Definition 6 is stated:

Remark 7. If $f \in \mathcal{A}, f(z)=z+a_{2} z^{2}+\ldots=z+\sum_{k=2}^{\infty} a_{k} z^{k}$, then

$$
L^{m}[f](z)=\frac{2}{z} \int_{0}^{z} L^{m-1}[f](t) d t=z+\sum_{k=2}^{\infty} \frac{2^{m}}{(k+1)^{m}} a_{k} z^{k}
$$

and

$$
z\left(L^{m}[f](z)\right)^{\prime}=2 L^{m-1}[f](z)-L^{m}[f](z), z \in U .
$$

We next give a definition and an important lemma from the admissible function method:

Definition 8. ([10, Definition 2.2b, p. 21]). We denote by $Q$ the set of functions $q$ that are analytic and injective on $\bar{U} \backslash E(q)$, where

$$
E(q)=\left\{\zeta \in \partial U: \lim _{z \rightarrow \zeta} q(z)=\infty\right\}
$$

and are such that $q^{\prime}(\zeta) \neq 0$ for $\zeta \in \partial U \backslash E(q)$. The set $E(q)$ is called exception set.

Lemma 9. $\boldsymbol{A}$ ([9, Lemma 2.2d, p. 24]). Let $q \in Q$ with $q(0)=a$ and let

$$
p(z)=z+a_{n} z^{n}+a_{n+1} z^{n+1}+\ldots
$$

be analytic in $U$, with $p(z) \neq a$ and $n \geq 1$. If $p$ is not subordinate to $q$, then there exist points $\tau_{0}=r_{0} e^{i \theta_{0}} \in U$ and $\zeta_{0} \in \partial U \backslash E(q)$, and an $p \geq n \geq 1$ for which $p\left(U r_{0}\right) \subset q(U)$,

(i) $p\left(\tau_{0}\right)=q\left(\zeta_{0}\right)$

(ii) $\tau_{0} p^{\prime}\left(\tau_{0}\right)=m \zeta_{0} q^{\prime}\left(\zeta_{0}\right)$ and

(iii) $\operatorname{Re}\left(\frac{\tau_{0} p^{\prime \prime}\left(\tau_{0}\right)}{p^{\prime}\left(\tau_{0}\right)}+1\right) \geq p \operatorname{Re}\left(\frac{\zeta_{0} q^{\prime \prime}\left(\zeta_{0}\right)}{q^{\prime}\left(\zeta_{0}\right)}+1\right)$.

In this paper, the original results are obtained by using the method of the subordination chains. The definition of the subordination chain and some important lemmas related to this method now follow.

Definition 10. ([10, p. 4]). A function $L(z, t), z \in U, t \geq 0$, is a subordination chain if $L(\cdot, t)$ is analytic and univalent in $U$, for all $t \geq 0$, and $L\left(z, t_{1}\right) \prec L\left(z, t_{2}\right)$, when $0 \leq t_{1}<t_{2}<\infty$.

Lemma 11. B ([10, p. 4]). The function

$$
L(z, t)=a_{1}(t) z+a_{2}(t) z^{2}+\ldots
$$

with $a_{1}(t) \neq 0$ for $t \geq 0$ and $\lim _{t \rightarrow \infty}\left|a_{1}(t)\right|=\infty$ is a subordination chain if and only if

$$
R e \frac{z \frac{\partial L(z, t)}{\partial z}}{\frac{\partial L(z, t)}{\partial t}}>0, \quad z \in U, t \geq 0 .
$$

Lemma 12. $C([10$, Theorem $3.44, p .132])$. Let $q$ be univalent in $U$ and let $\theta$ and $\phi$ be analytic in a domain $D$ containing $q(U)$, with $\phi(\omega) \neq 0$, when $\omega \in q(U)$. Set $Q(z)=z q^{\prime}(z) \phi[q(z)], h(z)=\theta[q(z)]+Q(z)$ and suppose that either

i) $h$ is convex or

ii) $Q$ is starlike.

In addition, assume that 
iii) $\operatorname{Re} \frac{z h^{\prime}(z)}{Q(z)}=\operatorname{Re}\left[\frac{\theta^{\prime}[q(z)]}{\phi[q(z)]}+\frac{z Q^{\prime}(z)}{Q(z)}\right]>0$.

If $p$ is analytic in $U$, with $p(0)=q(0), p(U) \subset D$ and

$$
\theta[p(z)]+z p^{\prime}(z) \phi[p(z)] \prec \theta[q(z)]+z q^{\prime}(z) \phi[q(z)]=h(z)
$$

then

$$
p(z) \prec q(z), z \in U
$$

and $q$ is the best dominant.

Lemma 13. $\boldsymbol{D}$ ([10, Theorem 3.16, $p$. 71. Hallenbeck and Ruscheweyh]). Let $h$ be a convex function with $h(0)=a$ and let

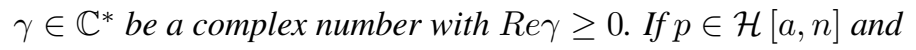

$$
p(z)+\frac{n}{\gamma} z p^{\prime}(z) \prec h(z), z \in U,
$$

then

$$
p(z) \prec q(z) \prec h(z), z \in U
$$

where

$$
q(z)=\frac{\gamma}{n z^{\frac{\gamma}{n}}} \int_{0}^{z} h(t) t^{\frac{\gamma}{n}-1} d t .
$$

The function $q$ is convex and is the best $(a, n)$-dominant.

A paper containing subordination results related to a class of univalent functions obtained by the use of an operator introduced by using a differential operator and an integral one has been published recently ([17]) and inspired the results from this paper.

\section{Main Results}

Firstly, we define a new differential-integral operator.

Definition 14. Let $0 \leq \lambda \leq 1, m \in \mathbb{N}$. Denote by $I^{m}: \mathcal{A} \rightarrow \mathcal{A}$, the differential-integral operator defined as

$$
I^{m}[f](z)=(1-\lambda) S^{m}[f](z)+\lambda L^{m}[f](z), z \in U
$$

Remark 15. a) For $\lambda=0$ obtain $I^{m}[f](z)=S^{m}[f](z)$, the differential operator Sălăgean [18].

b) For $\lambda=1$ obtain $I^{m}[f](z)=L^{m}[f](z)$, the integral operator Libera [6].

c) For $f \in \mathcal{A}, f(z)=z+\sum_{k=2}^{\infty} a_{k} z^{k}$, we have

$$
I^{m}[f](z)=z+\sum_{k=2}^{\infty}\left[k^{m}(1-\lambda)+\lambda \frac{2^{m}}{(k+1)^{m}}\right] a_{k} z^{k} .
$$

Next, we define a new class of univalent functions by using the newly introduced operator.

Definition 16. If $0 \leq \beta<1,0 \leq \lambda \leq 1, m \in \mathbb{N}$, we let $M(m, \beta, \lambda)$ stand for the class of functions $f \in \mathcal{A}$, which satisfy the inequality

$$
\operatorname{Re}\left(I^{m}[f](z)\right)^{\prime}>\beta, z \in U,
$$

where the differential-integral operator $I^{m}[f]$ is given by (2.1).

Remark 17. a) For $m=0, \beta=0,0 \leq \lambda \leq 1$, the operator $I^{m}[f]$ becomes

$$
I^{0}[f](z)=(1-\lambda) S^{0}[f](z)+\lambda R^{0}[f](z)=(1-\lambda) f(z)+\lambda f(z)=f(z), z \in U,
$$

then $M(m, \beta, \lambda)$ becomes

$$
M(0,0, \lambda)=R=\left\{f \in \mathcal{A}: \operatorname{Re}^{\prime}(z)>0, z \in U\right\}
$$

called the class of functions with bounded rotation.

This class of functions was studied by Alexander [1] and he proved that $R \subset \mathcal{S}$, Krzyz [4] and Mocanu [11] have proved that $R \not \subset \mathcal{S}^{*}$. A more systematic study of the class $R$ was done by Mac Gregor [6].

b) For $m=0,0 \leq \beta<1,0 \leq \lambda \leq 1$, we have

$$
R(\beta)=M(0, \beta, \lambda)=\left\{f \in \mathcal{A}: \operatorname{Re} f^{\prime}(z)>\beta\right\} \subset R=M(0,0, \lambda) .
$$


Theorem 18. The set $M(m, \beta, \lambda)$ is convex.

Proof. Let the functions

$$
f_{j}(z)=z+\sum_{k=2}^{\infty} \alpha_{k j} z^{j}, j=1,2, z \in U
$$

where $\alpha_{k j}=a_{k j}\left\{(1-\lambda) k^{m}+\lambda \frac{2^{m}}{(k+1)^{m}}\right\}$, be in the class $M(m, \beta, \lambda)$.

It is sufficient to show that the function

$$
h(z)=\mu_{1} f_{1}(z)+\mu_{2} f_{2}(z), z \in U,
$$

with $\mu_{1}, \mu_{2} \geq 0$ and $\mu_{1}+\mu_{2}=1$ is in $M(m, \beta, \lambda)$. Since $h(z)=z+\sum_{k=2}^{\infty}\left(\mu_{1} \alpha_{k 1}+\mu_{2} \alpha_{k 2}\right) z^{k}, z \in U$, then

$$
I^{m}[h](z)=z+\sum_{k=2}^{\infty}\left\{(1-\lambda) k^{m}+\lambda \frac{2^{m}}{(k+1)^{m}}\right\}\left(\mu_{1} a_{k 1}+\mu_{2} a_{k 2}\right) z^{k} .
$$

Differentiating (2.4), we get

$$
\left(I^{m}[h](z)\right)^{\prime}=1+\sum_{k=2}^{\infty}\left\{(1-\lambda) k^{m}+\lambda \frac{2^{m}}{(k+1)^{m}}\right\}\left(\mu_{1} a_{k 1}+\mu_{2} a_{k 2}\right) k z^{k-1} .
$$

Hence

$$
\begin{gathered}
\operatorname{Re}\left(I^{m}[h](z)\right)^{\prime}=1+\operatorname{Re} \sum_{k=2}^{\infty}\left[(1-\lambda) k^{m}+\lambda \frac{2^{m}}{(k+1)^{m}}\right] \mu_{1} a_{k 1} k z^{k-1} \\
+\operatorname{Re} \sum_{k=2}^{\infty}\left[(1-\lambda) k^{m}+\lambda \frac{2^{m}}{(k+1)^{m}}\right] \mu_{2} a_{k 2} k z^{k-1} .
\end{gathered}
$$

Since $f_{1}, f_{2} \in M(m, \beta, \lambda)$, we obtain

$$
\mu_{1} \operatorname{Re} \sum_{k=2}^{\infty}\left[(1-\lambda) k^{m}+\lambda \frac{2^{m}}{(k+1)^{m}}\right] \mu_{1} a_{k 1} k z^{k-1}>\mu_{1}(\beta-1),
$$

and

$$
\mu_{2} R e \sum_{k=2}^{\infty}\left[(1-\lambda) k^{m}+\lambda \frac{2^{m}}{(k+1)^{m}}\right] \mu_{2} a_{k 2} k z^{k-1}>\mu_{2}(\beta-1) .
$$

Using (2.6) and (2.7) in (2.5), we get

$$
\operatorname{Re}\left(I^{m}[h](z)\right)^{\prime}>1+\mu_{1}(\beta-1)+\mu_{2}(\beta-1)
$$

and $\mu_{1}+\mu_{2}=1$, we deduce $\operatorname{Re}\left(I^{m}[h](z)\right)^{\prime}>\beta, z \in U$, i.e. $M(m, \beta, \lambda)$ is convex.

Theorem 19. If $0 \leq \beta<1,0 \leq \lambda \leq 1$ and $m \in \mathbb{N}$, then we have

$$
M(m, \delta, \lambda) \subset M(m, \beta, \lambda),
$$

where

$$
\delta=2 \beta-1+2(1-\beta) \ln 2, \delta \approx 0,69 \beta+0,31<1 \text {. }
$$

The result is sharp.

Proof. If $\ln 2 \approx 0,69,0 \leq \beta<1,0 \leq 0,69 \beta<0,69,0,31 \leq 0,69 \beta+0,31<0,69+0,31,0,31 \leq 0,69 \beta+0,31<1$ and we have $\delta \approx 0,69 \beta+0,31<1$.

Let convex function

$$
h(z)=\frac{1+(2 \beta-1) z}{1+z}, z \in U .
$$

For $z \in U$, we have $\operatorname{Reh}(z)>\beta$, and $h(0)=1$. 
We let $f \in M(m, \beta, \lambda)$. From Definition 16, we have

$$
\operatorname{Re}\left(I^{m}[f](z)\right)^{\prime}>\beta, z \in U .
$$

Let

$$
p(z)=\frac{I^{m}[f](z)}{z}, z \in U
$$

Using (2.2) in (2.10), we have

$$
\begin{gathered}
p(z)=\frac{I^{m}[f](z)}{z}=\frac{z+\sum_{k=2}^{\infty}\left[(1-\lambda) k^{m}+\lambda \frac{2^{m}}{(k+1)^{m}}\right] a_{k} z^{k}}{z}= \\
1+\sum_{k=2}^{\infty}\left[(1-\lambda) k^{m}+\lambda \frac{2^{m}}{(k+1)^{m}}\right] a_{k} z^{k-1}=1+p_{1} z+p_{2} z^{2}+\ldots
\end{gathered}
$$

and $p(0)=1, p \in \mathcal{H}[1,1]$.

Differentiating (2.10), we get

$$
\left(I^{m}[f](z)\right)^{\prime}=p(z)+z p^{\prime}(z), z \in U
$$

Using (2.11) in (2.9), we have

$$
\operatorname{Re}\left(p(z)+z p^{\prime}(z)\right)>\beta, z \in U .
$$

Relation (2.12) can be written as a subordination of the form

$$
p(z)+z p^{\prime}(z) \prec h(z)+\frac{1+(2 \beta-1) z}{1+z} .
$$

Using Lemma 13 for $\gamma=1, n=1$, we have

$$
p(z) \prec q(z)=\frac{1}{z} \int_{0}^{z} h(t) d t=\frac{1}{z} \int_{0}^{z} \frac{(2 \beta-1) t+1}{t+1} d t=2 \beta-1+2(1-\beta) \frac{\ln (1+z)}{z} .
$$

The function $q$ is convex and is the best dominant.

Since $q$ is a convex function and $p(z) \prec q(z), z \in U$, we have

$$
\operatorname{Rep}(z)>q(1)=2 \beta-1+2(1-\beta) \ln 2=\delta .
$$

Usin(2.10) in (2.13), we have

$$
R e \frac{I^{m}[f](z)}{z}>\delta=2 \beta-1+2(1-\beta) \ln 2
$$

Using (2.2), we have

$$
\begin{gathered}
\frac{I^{m}[f](z)}{z}=\frac{z+\sum_{k=2}^{\infty}\left[(1-\lambda) k^{m}+\lambda \frac{2^{m}}{(k+1)^{m}}\right] a_{k} z^{k}}{z}= \\
1+\sum_{k=2}^{\infty}\left[(1-\lambda) k^{m}+\lambda \frac{2^{m}}{(k+1)^{m}}\right] a_{k} z^{k-1}= \\
1+\sum_{k=2}^{\infty}\left[(1-\lambda) k^{m}+\lambda \frac{2^{m}}{(k+1)^{m}}\right] k \frac{a_{k}}{k} z^{k-1}= \\
\left(z+\sum_{k=2}^{\infty}\left[(1-\lambda) k^{m}+\lambda \frac{2^{m}}{(k+1)^{m}}\right] b_{k} z^{k}\right)^{\prime}=\left(I^{m}[g](z)\right)^{\prime},
\end{gathered}
$$

where $g(z)=z+\sum_{k=2}^{\infty} b_{k} z^{k}, g \in \mathcal{A}$.

Using (2.14) in (2.15), we get

$$
\operatorname{Re} \frac{I^{m}[f](z)}{z}=\operatorname{Re}\left(I^{m}[g](z)\right)^{\prime}>\delta .
$$

Using Definition 16, we have $g \in M(m, \delta, \lambda)$.

Since $g \in M(m, \delta, \lambda)$ was taken arbitrary and we have proved that $f \in M(m, \beta, \lambda)$, we conclude that $M(m, \delta, \lambda) \subset$ $M(m, \beta, \lambda)$. 
From Theorem 19 we deduce the following corollary:

Corollary 20. If $f \in M(m, \delta, \lambda)$, then

$$
\operatorname{Re} \frac{I^{m}[f](z)}{z}>2 \beta-1+2(1-\beta) \ln 2=\delta .
$$

Proof. From the proof of Theorem 19, we can see that

$$
\frac{I^{m}[f](z)}{z} \prec q(z)=\frac{1}{z} \int_{0}^{z} h(t) d t=\frac{1}{z} \int_{0}^{z} \frac{1+(2 \beta-1) t}{1+t} d t=2 \beta-1+2(1-\beta) \frac{\ln (1+z)}{z} .
$$

Since $q$ is convex function, we have

$$
\operatorname{Re} \frac{I^{m}[f](z)}{z}>\operatorname{Req}(1)=2 \beta-1+2(1-\beta) \ln 2=\delta .
$$

Theorem 21. Let $q$ be univalent in $U$, and let $\theta$ and $\phi$ be analytic functions in a domain $D$ containing $q(U)$, with $\theta: \mathbb{C} \rightarrow \mathbb{C}$, $\theta(w)=w, \phi: \mathbb{C} \rightarrow \mathbb{C}, \phi(w)=\frac{1}{w}, \phi(w) \neq 0$, where $w \in q(U)$.

Set

$$
Q(z)=z q^{\prime}(z) \phi[q(z)], h(z)=\theta[q(z)]+Q(z)
$$

and suppose that we have

(i) $Q$ is starlike;

(ii) $\operatorname{Re} \frac{z h^{\prime}(z)}{Q(z)}=\operatorname{Re}\left[\frac{\theta^{\prime}[q(z)]}{\phi[q(z)]}+\frac{z Q^{\prime}(z)}{Q(z)}\right]>0, z \in U$.

If $p$ is analytic in $U$ with $p(0)=q(0), p(U) \subset D$, then

$$
\left(I^{m}[f](z)\right)^{\prime}+\frac{z\left(I^{m}[f](z)\right)^{\prime \prime}}{\left(I^{m}[f](z)\right)^{\prime}} \prec q(z)+\frac{z q^{\prime}(z)}{q(z)}
$$

implies

$$
\left(I^{m}[f](z)\right)^{\prime} \prec q(z), \quad z \in U
$$

and $q$ is the best dominant.

Proof. From (i) we know that the function $Q$ is starlike and from (ii) we know that the function $h$ is close-to-convex.

Let the function:

$$
\begin{gathered}
L(z, t)=a_{1}(t) z+a_{2}(t) z^{2}+\ldots=h(z)+t Q(z)= \\
\theta[q(z)]+(1+t) Q(z)=\theta[q(z)]+(1+t) z q^{\prime}(z) \phi[q(z)] .
\end{gathered}
$$

This function is analytic in $U$ for all $t \geq 0$ and is continuously differentiable on $[0, \infty)$ for $z \in U$.

Differentiating (2.18) with respect to $z$ we obtain

$$
\begin{gathered}
\frac{\partial L(z, t)}{\partial z}=a_{1}(t)+2 a_{2}(t) z+\ldots= \\
\theta^{\prime}[q(z)] q^{\prime}(z)+(1+t)\left\{q^{\prime}(z) \phi[q(z)]+z q^{\prime \prime}(z) \phi[q(z)]+z q^{\prime}(z) \phi^{\prime}[q(z)]\right\} .
\end{gathered}
$$

For $z=0$ we have

$$
a_{1}(t)=\theta^{\prime}[q(0)] q^{\prime}(0)+(1+t) q^{\prime}(0) \phi[q(0)]=q^{\prime}(0) \phi[q(0)]\left[\frac{\theta^{\prime}[q(0)]}{\phi[q(0)]}+1+t\right] \neq 0 .
$$

Differentiating (2.18) with respect to $t$ we obtain

$$
\frac{\partial L(z, t)}{\partial t}=Q(z)=z q^{\prime}(z) \phi[q(z)] .
$$

We deduced

$$
\operatorname{Re} \frac{z \frac{\partial L(z, t)}{\partial z}}{\frac{\partial L(z, t)}{\partial t}}=\operatorname{Re}\left[\frac{\theta^{\prime}(q(z))}{\phi[q(z)]}+(1+t) \frac{z Q^{\prime}(z)}{Q(z)}\right] .
$$


From (i) and (ii), $t \geq 0$, we obtain

$$
\operatorname{Re} \frac{z \frac{\partial L(z, t)}{\partial z}}{\frac{\partial L(z, t)}{\partial t}}=\operatorname{Re}\left[\frac{\theta^{\prime}(q(z))}{\phi[q(z)]}+\frac{z Q^{\prime}(z)}{Q(z)}\right]+t \operatorname{Re} \frac{z Q^{\prime}(z)}{Q(z)}>0 .
$$

Hence $a_{1}(t) \neq 0, \lim _{t \rightarrow \infty} a_{1}(t)=\infty$ and $\operatorname{Re} \frac{z \frac{\partial L(z z, t)}{\partial L(z, t)}}{\frac{\partial t}{\partial t}}>0$, for $z \in U$ and $t \geq 0$. Using Lemma $11, L(z, t)$ is a subordination chain which by Definition 10 implies

$$
L(z, s) \prec L(z, t) \text { for } 0 \leq s \leq t,(L(U, s) \subset L(U, t)) .
$$

For $t=0,(2.18)$ becomes

$$
L(z, 0)=\theta[q(z)]+z q^{\prime}(z) \phi[q(z)]=h(z), z \in U
$$

then (2.19) for $s=0$ becomes

$$
h(z) \prec L(z, t), t \geq 0, z \in U, h(U)=L(U, 0) \subset L(U, t) .
$$

Using (2.20) and Definition 10 we get

$$
L(\zeta, t) \notin h(U),|\zeta|=1, t \geq 0 .
$$

Let the function $\psi: \mathbb{C}^{2} \times \bar{U} \rightarrow \mathbb{C}$, and $\theta$ and $\phi$ be analytic functions

$$
\psi(r, s)=\theta(r)+s \phi(r), \theta(r)=r, \phi(r)=\frac{1}{r} .
$$

For $r=p(z), s=z p^{\prime}(z), z \in U$, we have

$$
\psi\left(p(z), z p^{\prime}(z)\right)=p(z)+z p^{\prime}(z) \frac{1}{p(z)}, \quad z \in U .
$$

For $r=q(z), s=z q^{\prime}(z), z \in U$, we have

$$
\psi\left(q(z), z q^{\prime}(z)\right)=q(z)+z q^{\prime}(z) \frac{1}{q(z)}, \quad z \in U .
$$

We let

$$
p(z)=\left(I^{m}[f](z)\right)^{\prime}, z \in U
$$

Using (2.2) and (2.24), we have

$$
\begin{gathered}
p(z)=\left(z+\sum_{k=2}^{\infty}\left[(1-\lambda) k^{m}+\lambda \frac{2^{m}}{(k+1)^{m}}\right] a_{k} z^{k}\right)^{\prime}= \\
1+\sum_{k=2}^{\infty}\left[(1-\lambda) k^{m}+\lambda \frac{2^{m}}{(k+1)^{m}}\right] k a_{k} z^{k-1}, p(0)=1, p \in \mathcal{H}[1,1] .
\end{gathered}
$$

Differentiating (2.24) and after short calculation, we obtain

$$
p(z)+\frac{z p^{\prime}(z)}{p(z)}=\left(I^{m}[f](z)\right)^{\prime}+\frac{z\left(I^{m}[f](z)\right)^{\prime \prime}}{\left(I^{m}[f](z)\right)^{\prime}}, z \in U .
$$

Using (2.22), (2.23) and (2.25), subordination (2.16) becomes

$$
\psi\left(p(z), z p^{\prime}(z)\right) \prec \psi\left(q(z), z q^{\prime}(z)\right), z \in U .
$$

In order to prove that (2.16) or (2.26) implies $p$ is subordinate to function $q$, we applying Lemma 9. For that we assume that the functions $p, q$ and $h$ satisfy the conditions in Lemma 9 in the unit disk $\bar{U}$.

Assume that function $p$ is not subordinate to function $q$. By Lemma 9, there exist points $z_{0}=r_{0} e^{i \theta_{0}} \in U$ anf $\zeta_{0} \in \partial U \backslash E(q)$ and $m \geq n \geq 1$, that satisfy

$$
p\left(z_{0}\right)=q\left(\zeta_{0}\right) \text { and } z_{0} p^{\prime}\left(z_{0}\right)=p \zeta_{0} q^{\prime}\left(\zeta_{0}\right)
$$


Then

$$
\psi\left(p\left(z_{0}\right), z_{0} p^{\prime}\left(z_{0}\right)\right)=\theta\left[p\left(z_{0}\right)\right]+z_{0} p^{\prime}\left(z_{0}\right) \phi\left[p\left(z_{0}\right)\right]=\theta\left[q\left(z_{0}\right)\right]+p \zeta_{0} q^{\prime}\left(\zeta_{0}\right) \phi\left[q\left(\zeta_{0}\right)\right] .
$$

If we take $t=p-1 \geq 0$, then

$$
L(z, p-1)=\theta[q(z)]+p z q^{\prime}(z) \phi[q(z)] .
$$

For $z=\zeta_{0} \in \partial U \backslash E(q)$, relation (2.28) becomes

$$
L\left(\zeta_{0}, p-1\right)=\theta\left[q\left(\zeta_{0}\right)\right]+p \zeta_{0} q^{\prime}\left(\zeta_{0}\right) \phi\left[q\left(\zeta_{0}\right)\right], \quad\left|\zeta_{0}\right|=1
$$

Using (2.27) and (2.29), we have

$$
L\left(\zeta_{0}, p-1\right)=\psi\left(p\left(z_{0}\right), z_{0} p^{\prime}\left(z_{0}\right)\right) \in h(U), z_{0} \in U
$$

Relation (2.30) contradicts (2.21), which proves that the assumption we made is false, hence $p$ is subordinate to $q$.

Since $q$ is the solution of the univalent equation

$$
h(z)=\theta[q(z)]+z q^{\prime}(z) \phi[q(z)], z \in U,
$$

we have that $q$ is the best dominant.

From Theorem 21 we deduce the following sufficient conditions for univalent function.

Criterion 22. Let

$$
q(z)=\frac{1+(2 \beta-1) z}{1+z}, 0 \leq \beta<1,
$$

be convex function with $q(0)=1$ and $\operatorname{Re} q(z)>\beta, z \in U$, and $q(z)+\frac{z q^{\prime}(z)}{q(z)}=\frac{1+(2 \beta-1) z}{1+z}+\frac{2(\beta-1) z}{(1+z)[1+(2 \beta-1) z]}, z \in U$.

If $f \in \mathcal{A}, m \in \mathbb{N}$ and satisfies the differential subordination

$$
\left(I^{m}[f](z)\right)^{\prime}+\frac{z\left(I^{m}[f](z)\right)^{\prime \prime}}{\left(I^{m}[f](z)\right)^{\prime}} \prec \frac{1+(2 \beta-1) z}{1+z}+\frac{2(\beta-1) z}{(1+z)[1+(2 \beta-1) z]},
$$

then

$$
\left(I^{m}[f](z)\right)^{\prime} \prec \frac{1+(2 \beta-1) z}{1+z}, z \in U,
$$

where $I^{m}[f]$ is defined by (2.1) and $f$ is an univalent function.

Proof. Since $q$ is convex, with $q(1)=\beta, 0 \leq \beta<1$, and $\operatorname{Re} q(z)>\beta$, relation (2.31) is equivalent to

$$
\operatorname{Re}\left(I^{m}[f](z)\right)^{\prime}>\operatorname{Re} q(1)=\beta .
$$

From Definition 16 we have $f \in M(m, \beta, \lambda)$ and $f$ is an univalent function.

Criterion 23. Let

$$
q(z)=\frac{1+z}{1-z}, \quad z \in U
$$

a convex function with $q(0)=1$ and $\operatorname{Re} q(z)>0, z \in U$, and $q(z)+\frac{z q^{\prime}(z)}{q(z)}=\frac{1+4 z+z^{2}}{1-z^{2}}, z \in U$.

If $f \in \mathcal{A}, m \in \mathbb{N}$ and satisfies the differential subordination

$$
\left(I^{m}[f](z)\right)^{\prime}+\frac{z\left(I^{m}[f](z)\right)^{\prime \prime}}{\left(I^{m}[f](z)\right)^{\prime}} \prec \frac{1+4 z+z^{2}}{1-z^{2}},
$$

then

$$
\left(I^{m}[f](z)\right)^{\prime} \prec \frac{1+z}{1-z}, \quad z \in U,
$$

where $I^{m}[f]$ is defined by (2.1) and $f$ is an univalent function.

Proof. Since $q$ is convex, with $q(1)=\beta, 0 \leq \beta<1$, and $\operatorname{Re} q(z)>0$, relation (2.32) is equivalent to

$$
\operatorname{Re}\left(I^{m}[f](z)\right)^{\prime}>\operatorname{Re} q(1)=0, z \in U .
$$

From Definition 16 we have $f \in M(0,0, \lambda)=R$ and $f$ is an univalent function. 
Example 24. Let $q(z)=\frac{1}{1-z}$ be an univalent function in $U$, with $q(0)=1$ and let the functions $\theta: \mathbb{C} \rightarrow \mathbb{C}$ and $\phi: \mathbb{C} \rightarrow \mathbb{C}$, $\theta(w)=w, \phi(w)=\frac{1}{w}, w \neq 0, w \in q(U)$.

If $w=q(z), \theta[q(z)]=q(z)=\frac{1}{1-z}, \phi[q(z)]=\frac{1}{q(z)}=1-z, z \in U$.

We calculate:

a) $\operatorname{Re} \frac{\theta^{\prime}[q(z)]}{\phi[q(z)]}=R e \frac{1}{1-z}>0, z \in U$.

b) $\operatorname{Re}\left(1+\frac{z q^{\prime \prime}(z)}{q^{\prime}(z)}-\frac{z q^{\prime}(z)}{q(z)}\right)=\operatorname{Re}\left(1+\frac{2 z}{1-z}-\frac{2}{1-z}\right)=\operatorname{Re} \frac{1}{1-z}>0$.

c) $h(z)=\theta[q(z)]+Q(z)=q(z)+\frac{z q^{\prime}(z)}{q(z)}=\frac{1}{1-z}+\frac{z}{1-z}=\frac{1+z}{1-z}$.

d) $p(0)=q(0)=1$.

For $f \in \mathcal{A}, f(z)=z+\frac{1}{4} z^{2}+\frac{1}{7} z^{3}, \lambda=\frac{1}{4}, m=2$, we have $I^{2}[f(z)]=z+\frac{7}{9} z^{2}+\frac{109}{112} z^{3}$.

$$
\left(I^{2}[f](z)\right)^{\prime}+\frac{z\left(I^{2}[f](z)\right)^{\prime \prime}}{\left(I^{2}[f](z)\right)^{\prime}}=1+\frac{14}{9} z+\frac{327}{112} z^{2}+\frac{1564 z+5886 z^{2}}{1008+9568 z+2943 z^{2}}
$$

and

$$
p(z)=\left(I^{2}[f](z)\right)^{\prime}=1+\frac{14}{9} z+\frac{327}{112} z^{2} .
$$

From Theorem 21, we have

$$
1+\frac{14}{9} z+\frac{327}{112} z^{2}+\frac{1564 z+5886 z^{2}}{1008+9568 z+2943 z^{2}} \prec \frac{1+z}{1-z}, \quad z \in U
$$

implies

$$
1+\frac{14}{9} z+\frac{327}{112} z^{2} \prec \frac{1}{1-z}, z \in U
$$

and $q(z)=\frac{1}{1-z}$ is the best dominant.

\section{Conclusion.}

A new differential-integral operator is introduced in this paper and using it, a new class of univalent functions is defined and studied. It is proved that the newly introduced class is convex and using the subordination chains method, theorems and corollaries related to Briot-Bouquet differential subordinations regarding the differential-integral operator introduced in the paper could be stated and proved. The best dominant for the Briot-Bouquet differential subordination is also provided. An example is incorporated in the paper too, which makes the results more interesting. The part which could be of most interest for future work are the two criteria for univalent functions stated and proved at the end of the paper.

\section{REFERENCES}

[1] J.W. Alexander, Functions which map the interior of the unit circle upon simple regions, Anals of Math., 17 (1915), $12-22$.

[2] T. Bulboacă, Differential subordinations and superodinations. Recent results, Casa Cărţii de Ştiinţă Cluj-Napoca, 2005.

[3] D.J. Hallenbeck, St. Ruscheweyh, Subordination by convex functions, Proc. Amer. Math. Soc., 52 (1975), 191-195.

[4] J. Krzyz, A counterexample concerning univalent functions, Folia Soc. Scient Lubliniensis, 2 (1962), 57-58.

[5] E. Hille, Ordinarry Differential Equations in the complex Plane, John Wiley, New York, 1976.

[6] R.J. Libera, Some classes of regular univalent functions, Proc. Amer. Math. Soc., 16 (1965), 755-758.

[7] T.H. Mac Gregor, Functions whose derivative has a positive real part, Trans. Amer. Math. Soc., 104 (1962), $532-537$.

[8] S.S. Miller and P.T. Mocanu, Second order differential inequalities in the complex plane, J. Math. Anal. Appl., 65 (1978), 298-305.

[9] S.S. Miller and P.T. Mocanu, Differential subordinations and univalent functions, Michig. math. J., 28 (1981), $157-171$.

[10] S.S. Miller and P.T. Mocanu, Differential Subordinations. Theory and Applications, Marcel Dekker Inc., New York, Basel, 2000.

[11] P.T. Mocanu, Some starlikeness conditions for analytic functions, Rev. Roum. Math. Pures Appl., 33, (1-2) (1988), 117-124.

[12] P.T. Mocanu, T. Bulboacă and G.S. Sălăgean, Geometric Function Theory, (Romanian), Casa Cărţii de Ştiinţă Cluj-Napoca, 1999. 
[13] G.I. Oros, Gh. Oros and R. Diaconu, Differential subordination obtained with some new integral operator, J. Computational Analysis and Applications, 19(5) (2015), 904-910.

[14] G.I. Oros, New differential subordinations and superordinations. Strong differential subordination. Strong differential superordination, Lambert Academic Publishing, Saarboucken, Germany, 2011.

[15] G.I. Oros, Using differential subordinations in the study of certain classes of univalent functions, (Romanian), Casa Cărţii de Ştiinţă Cluj-Napoca, 2008.

[16] Gh. Oros, Convexity and starlikeness in geometric function theory, Certaken by the PAMM - Centre at the Bute, Budapest, 2001.

[17] A.O. Pall-Szabo, On a class of univalent functions defined by Sălăgean integro-differential operator, Miskolc Mathematical Notes, 19 (2) (2018), 1095-1106.

[18] G.S. Sălăgean, Subclasses of univalent functions, Lectures Notes in Math, Springer-Verlag, 1013 (1983), $362-372$. 